\title{
Comparison of Catalyzing Properties of Bacterial 4-a-Glucanotransferases Focusing on Their Cyclizing Activity
}

\author{
Jung-Eun Kim ${ }^{1 \dagger}$, Phuong Lan Tran ${ }^{1,2,3 \dagger}$, Jae-Min Ko ${ }^{1}$, Sa-Rang Kim ${ }^{4}$, Jae-Han Kim ${ }^{4}$, and \\ Jong-Tae Park ${ }^{1 *}$ \\ ${ }^{1}$ Department of Food Science and Technology, Chungnam National University, Daejeon 34734, Republic of Korea \\ 2Department of Food Technology, An Giang University, An Giang, Vietnam \\ ${ }^{3}$ Vietnam National University, Ho Chi Minh, Vietnam \\ ${ }^{4}$ Department of Food Nutrition, Chungnam National University, Daejeon 34734, Republic of Korea
}

A newly cloned 4-a-glucanotransferase (aGT) from Deinococcus geothermalis and two typical bacterial aGTs from Thermus scotoductus and Escherichia coli (MaIQ) were investigated. Among 4 types of catalysis, the cyclization activity of aGTs that produces cycloamylose (CA), a valuable carbohydrate making inclusion complexes, was intensively studied. The new aGT, DgaGT, showed close protein sequence to the aGT from $T$. scotoductus (TsaGT). MalQ was clearly separated from the other two aGTs in the phylogenetic and the conserved regions analyses. The reaction velocities of disproportionation, cyclization, coupling, and hydrolysis of three aGTs were determined.

Intriguingly, MalQ exhibited more than 100-fold lower cyclization activity than the others. To lesser extent, the disproportionation activity of MalQ was relatively low. DgaGT and TsaGT showed similar kinetics results, but TsaGT had nearly 10-fold lower hydrolysis activity than DgaGT. Due to the very low cyclizing activity of MaIQ, DgaGT and TsaGT were selected for further analyses. When amylose was treated with DgaGT or TsaGT, CA with a broad DP range was generated immediately. The DP distribution of CA had a bimodal shape (DP 7 and 27 as peaks) for the both enzymes, but larger DPs of CA quickly decreased in the DgaGT. Cyclomaltopentaose, a rare cyclic sugar, was produced at early reaction stage and accumulated as the reactions went on in the both enzymes, but the increase was more profound in the TsaGT. Taken together, we clearly demonstrated the catalytic differences between aGT groups from thermophilic and pathogenic bacteria that and showed that aGTs play different roles depending on their lifestyle.

Keywords: 4-a-Glucanotransferase, cycloamylose, cyclization, disproportionation, cyclic maltopentaose

Received: September 10, 2020 Accepted: October 6, 2020

First published online: October 8, 2020

${ }^{*}$ Corresponding author Phone: +82-42-821-6728 Fax: +82-42-821-8785 E-mail: jtpark@cnu.ac.kr

'These authors contributed equally to this work.

pISSN 1017-7825 elSSN 1738-8872

Copyright(C) 2021 by The Korean Society for Microbiology and Biotechnology

\section{Introduction}

4- $\alpha$-Glucanotransferase ( $\alpha$ GT, E.C. 2.4.1.25) has intermolecular and intramolecular transglycosylation activities and is involved in cleaving $\alpha-1,4$-glycosidic linkages and generating new $\alpha$-1,4-linkages. This type of enzyme is widely distributed in bacteria, and similar enzymes are reported from some plants such as Solanum tubersum [14]. Thermostable aGTs from Thermus species were intensively studied for decades, and some Thermus aGTs were successfully applied to modifications of starch or starch derivatives [5-8]. For example, thermo-reversible starch gel was made of clusters that were generated by $\alpha \mathrm{GT}$ treatment on amylopectin [5]. On the other hand, MalQ, an aGT-type enzyme in many pathogenic bacteria, has been investigated to elucidate its role in the carbohydrate metabolism and pathogenesis of bacteria [9-11]. Esherichia coli MalQ has crucial roles in maltose/maltodextrin metabolism. MalQ is the only enzyme that utilizes maltose in E. coli [12]. Recently, we clearly demonstrated its role in the degradation of glycogen [1].

Catalytic reactions that are conducted by aGTs are classified into 4 types, disproportionation, cyclization, coupling, and hydrolysis [13]. Usually, the three transglycosylation activities are much stronger than the hydrolysis in common bacterial $\alpha$ GTs. When $\alpha$ GT cleaves an $\alpha-1,4$-glycosidic bond and transfers the donor glycan in the $\alpha-1,4$-bond to the non-reducing end of another glucan, that is the disproportionation reaction. The coupling reaction is different from the disproportionation, because it uses cyclic glucans as a donor molecule. Cyclization is an intramolecular transglycosylation. Well-known commercial products produced by this cyclization activity are cyclodextrins (CDs) [3,13].

Cycloamylose (CA) is a cyclic oligosaccharide composed of $\alpha$-1,4-glycosidic linkages, exclusively. Low degrees of polymerization (DPs) ranging from DP $6 \sim 8$ result in $\alpha-, \beta$-, and $\gamma$-CD, respectively. Generally, the terminology 
regarding CA includes broad DP ranges from small CDs to large molecules having DPs above 50. CDs have a hydrophobic central cavity and accommodate various guest molecules that form an inclusion complex and change their solubility, reactivity, or stability. CA, which contains cyclic glucans with inner cavities of various size, is also used as a host molecule for inclusion complex and protein refolding agent $[14,15]$.

Many aGTs have been reported but no research on the kinetic analyses of four different types of catalysis (disproportionation, cyclization, coupling, and hydrolysis) has been published. Therefore, in this study, we determined the reaction velocities of four types of catalysis using three bacterial $\alpha \mathrm{GTS}$ including a newly cloned aGT from Deinococcus geothermalis and well-characterized aGTs from T. scotoductus and E. coli. Assay methods for precise, direct detection of each catalysis type were successfully developed and applied. In addition, our intensive analyses on the cyclization reaction and CA production will provide valuable information for further practical research using aGTs.

\section{Materials and Methods \\ Protein Sequence Analysis}

Protein sequence analysis was carried out using the BLAST sequence analysis tool as described by Altschul et al. [16] with the gi number of each microorganism as follows: gi|38505481|_Thermus aquaticus, gi|570754797|_Thermus thermophilus, gi|320150052|_T. scotoductus, gi|310689644|_Thermus brockianus, gi|94555055|_D. geothermalis, gi|653292780|_Deinococcus radiodurans, gi|490649772|_Geobacter metallireducens, gi|18159937|_Pyrobaculum aerophilum, gi|446993535|_Vibrio cholerae, gi|647458543|_Vibrio parahaemolyticus, gi|695739988|_Enterobacter cloacae, gi|740849651|_Citrobacter freundii, gi|82778768|_Shigella dysenteriae, gi|16131292|_E. coli, gi|110616799|_Shigella flexneri.

\section{Expression and Purification of aGT Enzymes}

Escherichia coli MC1061 and BL21, the transformant hosts carrying the recombinant plasmids, were cultured overnight in LB medium containing ampicillin $(100 \mu \mathrm{g} / \mathrm{ml})$ at $37^{\circ} \mathrm{C}$ with shaking. After harvesting, the cell pellets were resuspended in lysis buffer [ $50 \mathrm{mM}$ Tris- $\mathrm{HCl}(\mathrm{pH} 7.5), 300 \mathrm{mM} \mathrm{NaCl}, 10 \mathrm{mM}$ imidazole] and then sonicated in an ice bath using sonicator (sonicator ultrasonic processor, Osonica, USA, $2.5 \mathrm{~min} \times 4$ times). The crude cell extracts were purified by FPLC Ni-NTA affinity chromatography with Ni-NTA resin (Qiagen, USA) packed in a column (Bio-Rad, USA). The purity and activity of the enzymes were determined by SDS-PAGE and Lugol's method, respectively $[8,17]$.

\section{Determination of Velocity of Four Types of aGT Reaction Types}

The disproportionation and coupling activities of the aGTs were assayed by the decrease of glucose amount using the glucose oxidase-peroxidase (GOPOD) method [18]. The reaction mixtures were prepared with $0.5 \%(\mathrm{w} / \mathrm{v})$ amylose and $0.025 \%(\mathrm{w} / \mathrm{v})$ glucose for disproportionation and $0.5 \%(\mathrm{w} / \mathrm{v})$ cycloamylose and $0.02 \%(\mathrm{w} / \mathrm{v})$ glucose for coupling in $50 \mathrm{mM}$ sodium acetate buffer (optimum pH 7.5 and 6.5 for TsaGT and both DgaGT and MalQ, respectively). They were separately incubated with $100 \mu$ l of each enzyme at optimum temperature of $37^{\circ} \mathrm{C}, 55^{\circ} \mathrm{C}$ and $75^{\circ} \mathrm{C}$ for MalQ, DgaGT and TsaGT, respectively. After cooling down, a $100 \mu$ aliquot of each reaction was mixed with $1 \mathrm{ml}$ of GOPOD solution, incubated at $37^{\circ} \mathrm{C}$ for $30 \mathrm{~min}$ and then the absorbance was measured at $510 \mathrm{~nm}$. The disproportionation or coupling activities of enzymes were defined as the amount of enzyme that can decrease $1 \mu$ mole of glucose per min.

The cyclization activity of the aGTs was measured by the increase of cycloamylose product using highperformance anion exchange chromatography (HPAEC) [19]. The reaction mixtures containing $0.5 \%(\mathrm{w} / \mathrm{v})$ amylose in $50 \mathrm{mM}$ sodium acetate buffer using optimum $\mathrm{pH}$ as described above were severally incubated with $100 \mu \mathrm{l}$ of each enzyme at optimum temperature as also described above. After enzyme reaction, the $100 \mu \mathrm{l}$ aliquots of the reaction mixtures were then treated with $\beta$-amylase to degrade linear maltooligosaccharides into maltose. The $\beta$-amylase-resistant cyclic glucans were isolated by ethanol precipitation and analyzed by HPAEC. The cyclization activity of enzymes was defined as the rate at which a certain enzyme amount can synthesize cycloamylose per min.

The hydrolysis activity of the aGTs was determined by the increase of reducing sugar using the copperbicinchoninate method [20]. The reaction mixtures containing $0.5 \%(\mathrm{w} / \mathrm{v})$ amylose in $50 \mathrm{mM}$ sodium acetate buffer at optimum $\mathrm{pH}$ were incubated with $100 \mu \mathrm{l}$ of each enzyme at optimum temperature. The enzyme reactions were eliminated by a same sample volume of $0.1 \mathrm{~N} \mathrm{NaOH}$. Then, $200 \mu$ laliquot of each enzyme reaction mixture was added with an equal amount of copper-bicinchoninate reagent and incubated at $80^{\circ} \mathrm{C}$ for $35 \mathrm{~min}$ and at which a certain enzyme amount at $575 \mathrm{~nm}$. The hydrolysis activity of enzymes was defined as the rate at which a certain enzyme amount can hydrolyze the substrate into reducing end sugar per min.

\section{Production of CA}

To produce CA, $0.1 \%(\mathrm{w} / \mathrm{v})$ amylose was separately incubated with $0.05 \mathrm{U} / \mathrm{mg}$ of purified DgaGT and TsaGT in $50 \mathrm{mM}$ sodium acetate buffer at optimum temperature. The reaction mixtures were treated with $\beta$-amylase to remove small maltodextrins. The CA products were then isolated by ethanol precipitation and centrifugation. The CA pellets were dried and resuspended in distilled water for further analysis.

\section{High-Performance Anion-Exchange Chromatography (HPAEC) Analysis}

The side chain distribution of modified starch was determined by HPAEC. The enzyme reaction mixtures were stopped by boiling for $5 \mathrm{~min}$ and centrifuged at $13,000 \times g$ and $4^{\circ} \mathrm{C}$ for $1 \mathrm{~min}$. The supernatants were filtered 
through $0.45 \mu \mathrm{m}$ membranes and then injected into the HPAEC system (DX-500 system, Dionex, USA) with a pulsed electrochemical detector (ED40, Dionex). The ingredients of samples were separated in a CarboPac PA1 column $(250 \times 4 \mathrm{~mm}$, Dionex) at a flow rate of $1 \mathrm{ml} / \mathrm{min}$ with a $10-64 \%(\mathrm{v} / \mathrm{v})$ gradient of $600 \mathrm{mM}$ sodium acetate in $150 \mathrm{mM} \mathrm{NaOH}$.

Matrix-Assisted Laser Desorption/Ionization Time-of-Flight/Mass Spectroscopy (MALDI-TOF/MS) Analysis

MALDI-TOF/MS analysis of cycloamylose products was carried out in positive ion reflectron mode using an UltrafleXtreme system (Bruker Daltonics, USA). One microliter of cycloamylose solution was spotted onto a stainless steel target plate and followed by $0.3 \mu \mathrm{l}$ of $0.01 \mathrm{M} \mathrm{NaCl}$ and $0.5 \mu \mathrm{l}$ of $5 \% 2,5$-dihydroxybenzoic acid in $50 \%$ acetonitrile. The spot was rapidly dried under vacuum for more homogenous crystallization. Mass spectra were externally calibrated using maltooligosaccharides isolated from commercial beer. Raw MS data was processed with FlexAnalysis software (version 3.3, Bruker Daltonics) [21].

\section{Results and Discussion}

\section{Sequence Analysis of Three aGTs}

Protein sequences of DgaGT, TsaGT, and MalQ were compared by conducting phylogenetic tree analysis with other aGTs from thermophilic or pathogenic bacteria (Fig. 1A). DgaGT showed the primary structure of the enzyme similar to the cluster of aGTs from genus Thermus. In contrast, MalQ was completely separated from the other two enzymes and clustered with aGTs from Shigella species. Intriguingly, the sequences from E. coli and S. flexneri aGTs are nearly identical (99.4\%, determined by BLAST provided by www.blast.ncbi.nlm.nih.gov). The phylogenetic tree was roughly divided into two big clusters of proteins: one group from thermophilic bacteria and the other from pathogenic bacteria. MalQ exhibits very high sequence homology with other aGTs from pathogenic bacteria, such as $S$. dysenteriae, C. freundii, and En. cloacae. In analysis of four well-conserved regions, aGTs from E. coli, S. flexneri, and En. cloacae have exactly the same sequences at all four regions (Fig. 1B). Among genus Thermus, the aGT sequences are strongly conserved by comparison with other carbohydrate enzymes such as glycogen branching enzymes [22].

It is plausible that horizontal aGT gene transfer happened among the pathogenic bacteria. In addition, the aGT sequences from Thermus were no different from their common ancestor. The conservation of the protein sequences strongly indicates a crucial role of $\alpha$ GTs in those bacteria. The role of $\alpha \mathrm{GT}$ in the thermophilic bacteria is probably different from that for the pathogens due to the profound differences in their lifestyles. In the next section, this postulation was proved by intensive analyses of the 4 types of catalysis conducted by the 3 aGTs.

\section{Determination Kinetic Parameters of Four Types of Catalysis}

The disproportionation, cyclization, coupling, and hydrolysis activities of the aGTs were determined using more quantitative methods than conventional ones. The assays were designed to quantitatively measure the number of catalytic reactions by determining increase of products or decrease of reactants. Basically, saturation kinetics were conducted to determine reaction velocities. The reaction mechanisms of the four types of catalysis and scheme of the detection methods are shown in Fig. 2. The detailed conditions are given in the Materials and Methods section.

Overall, three enzymes had strong activity on intermolecular tranglycosylation (disproportionation, coupling)

A

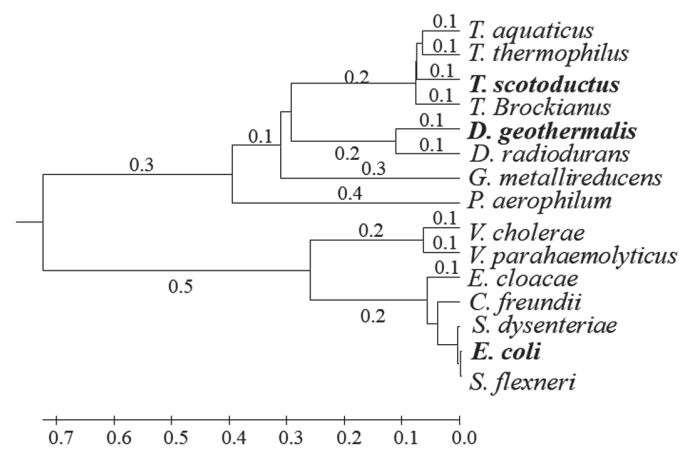

B

\begin{tabular}{lcc}
\hline & Region 1 & region 2 \\
\hline Deinococcus geothermalis & RGVGVIGDIPIFV & YDLIRIDHFRG \\
Thermus scotoductus & LGLSLIGDMPIFV & FHLVRIDHFRG \\
Thermus aquaticus & LGIQIIGDMPIFV & FHLVRVDHFRG \\
Thermus thermophilus & LGIRIIGDMPIFV & FHLVRIDHFRG \\
Deinococcus radiodurans & RGVQIIGDIPIFV & YDIIRVDHFRG \\
Escherichia coli & MPIGLYRDLAVGV & CGALRIDHVMS \\
Shigellaflexneri & MPIGLYRDLAVGV & CGALRIDHVMS \\
Enterobacter cloacae & MPIGLYRDLAVGV & CGALRIDHVMS \\
\hline \hline & region 3 & region 4 \\
\hline Deinococcus geothermalis & MPIIAEDLG & -QVVYTGTHD \\
Thermus scotoductus & VPILAEDLG & RVVVYTGTHD \\
Thermus aquaticus & VPILAEDLG & RVVVYTGTHD \\
Thermus thermophilus & VPVLAEDLG & RVVVYTGTHD \\
Deinococcus radiodurans & LPIIAEDLG & -RVVYTGTHD \\
Escherichia coli & CMVIGEDLG & -SMAVAATHD \\
Shigella flexneri & CMVIGEDLG & -SMAVAATHD \\
Enterobacter cloacae & CMVIGEDLG & -SMAVATTHD \\
\hline
\end{tabular}

Fig. 1. Phylogenic tree of $4 a G T$ protein sequences (A). The tree was built by the neighbor-joining method and implemented in the CLUSTALW program. Comparison of amino acid sequences in the 4 conserved regions of various $4 a \mathrm{GTS}(\mathrm{B})$. 
A Disproportionatio

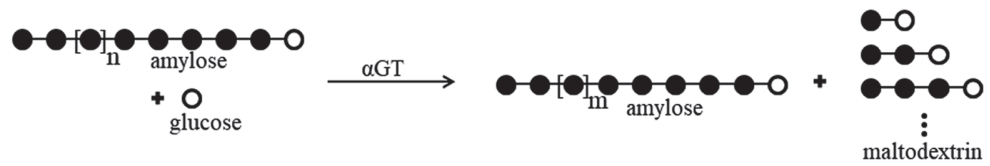

B Cyclization
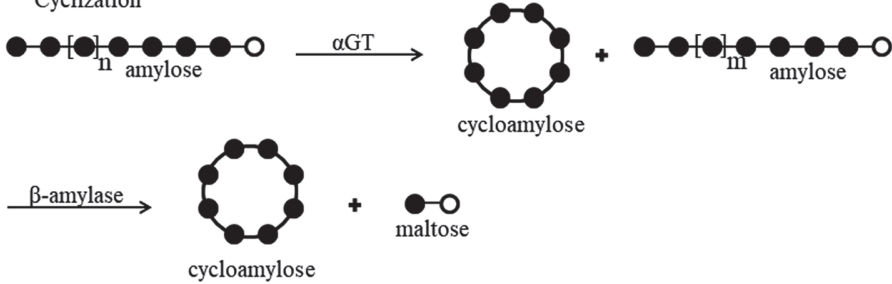

C Couplin

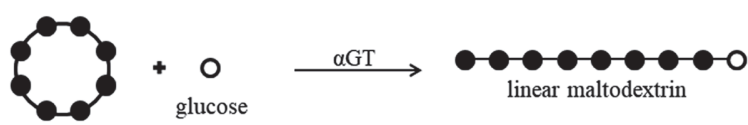

cycloamylose

D Hydrolysis

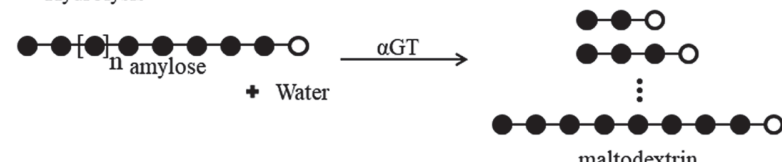

maltodextrin

Fig. 2. Schematics of the four reactions catalyzed by $4 \alpha$ GT. (A) Disproportionation: the enzyme cleaves and transfers the $\alpha$-1,4-linkages of saccharides to acceptor molecule. Amylose is used as the donor molecule and glucose as the acceptor molecule. (B) Cyclization: the non-reducing end of saccharides is used as the acceptor resulting in circular saccharides with $\alpha$ 1,4-linkages, cycloamyloses. (C) Coupling: the cycloamylose ring is cleaved and transferred to a glucose, the acceptor molecule, then linear product is generated. The cycloamylose is used as the donor, and the glucose as the acceptor. (D) Hydrolysis: a water molecule replaces the saccharide and shorter saccharides are produced. The black and white circles indicate the anhydroglucose residues and the reducing end glucoses, respectively.

compared with intramolecular reaction (cyclization) (Fig. 3, Table 1). Also, the hydrolysis activity was at least 1,000-fold lower than the tranglycosylation activities in all the enzymes. DgaGT and TsaGT exhibited similar catalytic properties, but TsaGT had about 5-fold greater transglycosylation/hydrolysis ratio. MalQ, which was completely separated from the others in the phylogenetic tree, showed extremely low activity for the cyclization. The coupling and the hydrolysis activities of MalQ were comparable to those of DgaGT, but the enzyme had disproportionation activity below one twelfth that of DgaGT. The results clearly indicate that aGTs from thermophilic bacteria, DgaGT and TsaGT, differently contribute to the maltose/maltodextrin metabolism from MalQ in E. coli [12].

When the activities are expressed relatively to the cyclization, the differences between the two aGT groups are

Table 1. The reaction velocities for 4 types of catalysis were compared as unit per nmole of protein.

\begin{tabular}{lccccc}
\hline \multicolumn{5}{c}{ Reaction velocity (U/nmole protein) } \\
\hline & Disproportionation & Cyclization & Coupling & Hydrolysis & Transglycosylation/Hydrolysis \\
\hline DgaGT & 3.75 & $2.21 \times 10^{-2}$ & 2.83 & $1.56 \times 10^{-3}$ & $4.23 \times 10^{3}$ \\
TSaGT & 3.43 & $3.79 \times 10^{-2}$ & 4.18 & $3.55 \times 10^{-4}$ & $2.15 \times 10^{4}$ \\
MalQ & 0.30 & $1.66 \times 10^{-4}$ & 1.92 & $1.10 \times 10^{-3}$ & $2.01 \times 10^{3}$ \\
\hline \multicolumn{5}{c}{ Relative activity to cyclization } \\
\hline \multicolumn{7}{c}{ Cyclization } & Coupling & $7.05 \times 10^{-2}$ \\
\hline DgaGT & Disproportionation & 1 & $1.28 \times 10^{2}$ & $9.36 \times 10^{-3}$ \\
TSaGT & $1.70 \times 10^{2}$ & 1 & $1.10 \times 10^{2}$ & 6.67
\end{tabular}

Relative values to cyclization activity are also given in a lower table. For the relative activities, comparison of the values from different enzymes are not proper. 

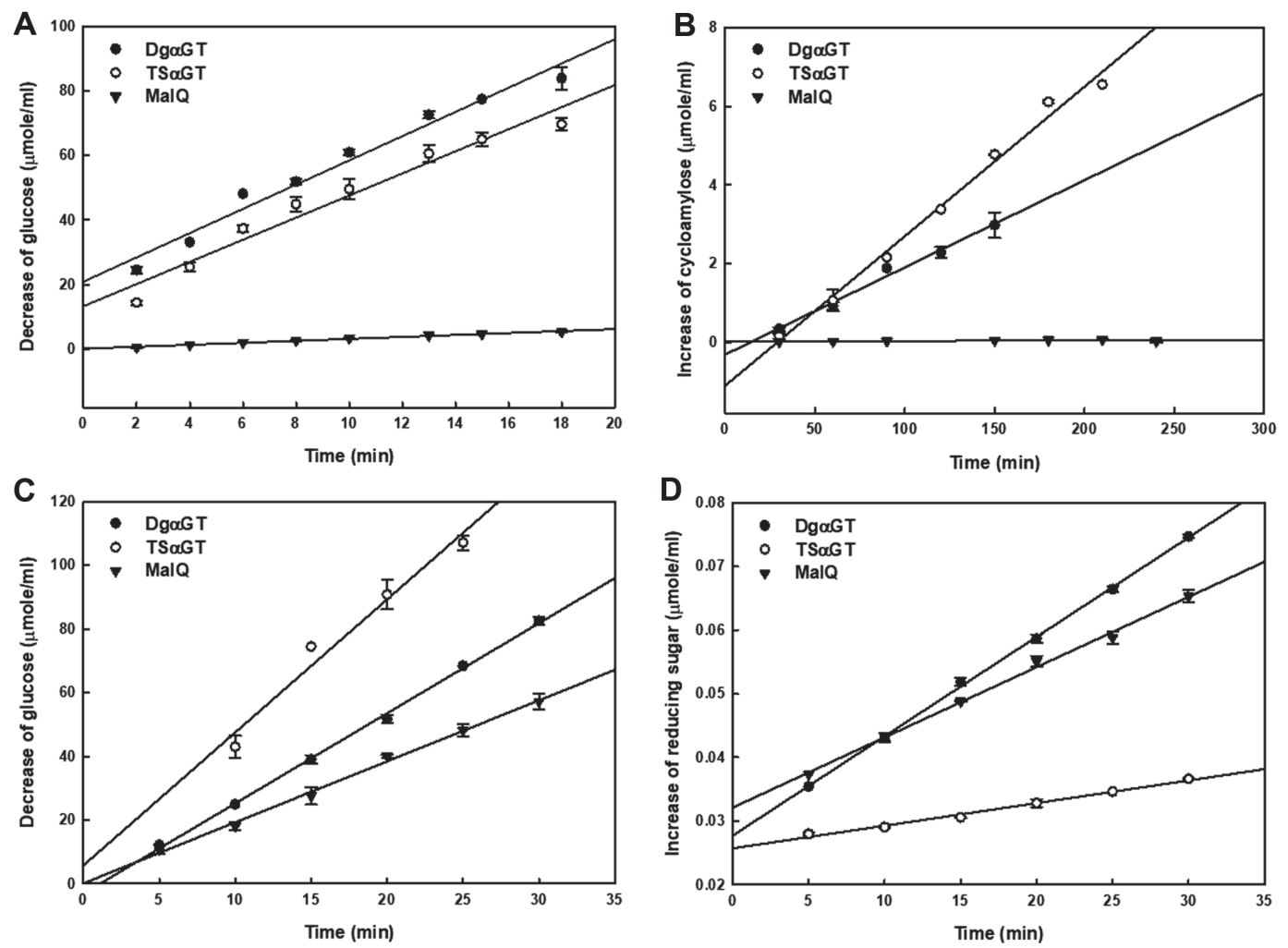

Fig. 3. Determination of reaction velocity for the disproportionation (A), the cyclization (B), the coupling (C), and the hydrolysis (D). Note that $\mathrm{x}$-y axis of all graphs has the same unit.

more prominent. In the E. coli-type lifecycle, cycloamylose probably has no meaningful role. Even though $\beta$-CD was detected from $E$. coli cell lysate (data not shown), the concentration was very low compared with other linear maltodextrins. The aGTs from thermophilic bacteria accumulated a greater amount of CA, when amylose or maltodextrin with a long DP was used as a substrate [7]. It is plausible that the enzymes accumulate CAs, and the cyclic glucans are recycled depending on bacterial metabolic phase. The accumulation of CAs in the cytosol of thermophilic bacteria can help with folding of proteins [23, 24]. In a practical approach, TsaGT might be a very good enzyme to produce CA. The enzyme has very low hydrolysis activity. However, relatively high coupling activity can disturb the accumulation of CA in high concentration. To solve this problem, one could try to continuously remove small maltodextrins that serve as receptors in the coupling reaction.

\section{Cyclization Activity and Cycloamylose Analyses}

To further investigate the cyclization properties of the aGTs, long-term reactions using amylose as a substrate were conducted for DgaGT and TsaGT. MalQ had too low cyclization activity to be used. The cyclization reaction

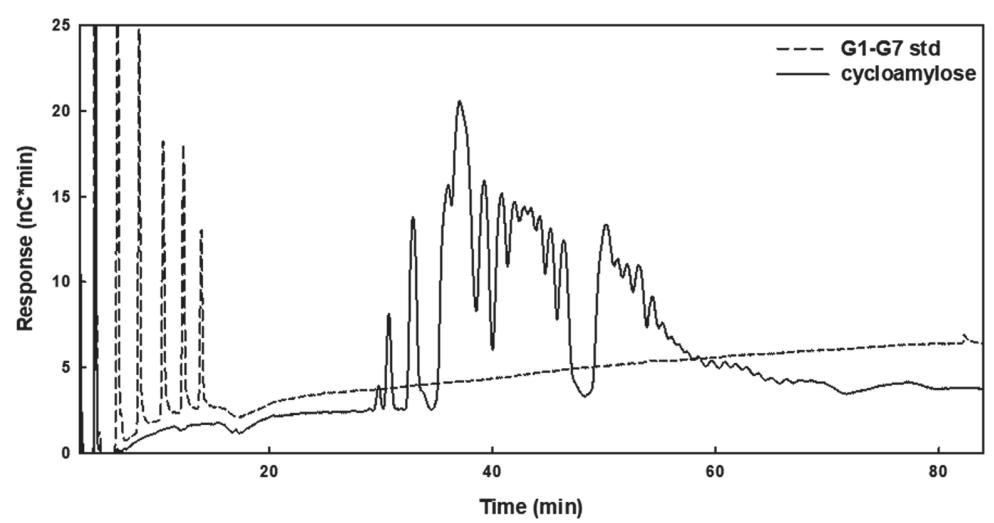

Fig. 4. Cycloamylose analysis produced by DgaGT using amylose as a substrate. At maximum yield of cycloamylose production ( $6 \mathrm{~h}$ reaction), the reactant was treated by $\beta$-amylase to remove linear saccharides, then analyzed by HPAEC. 

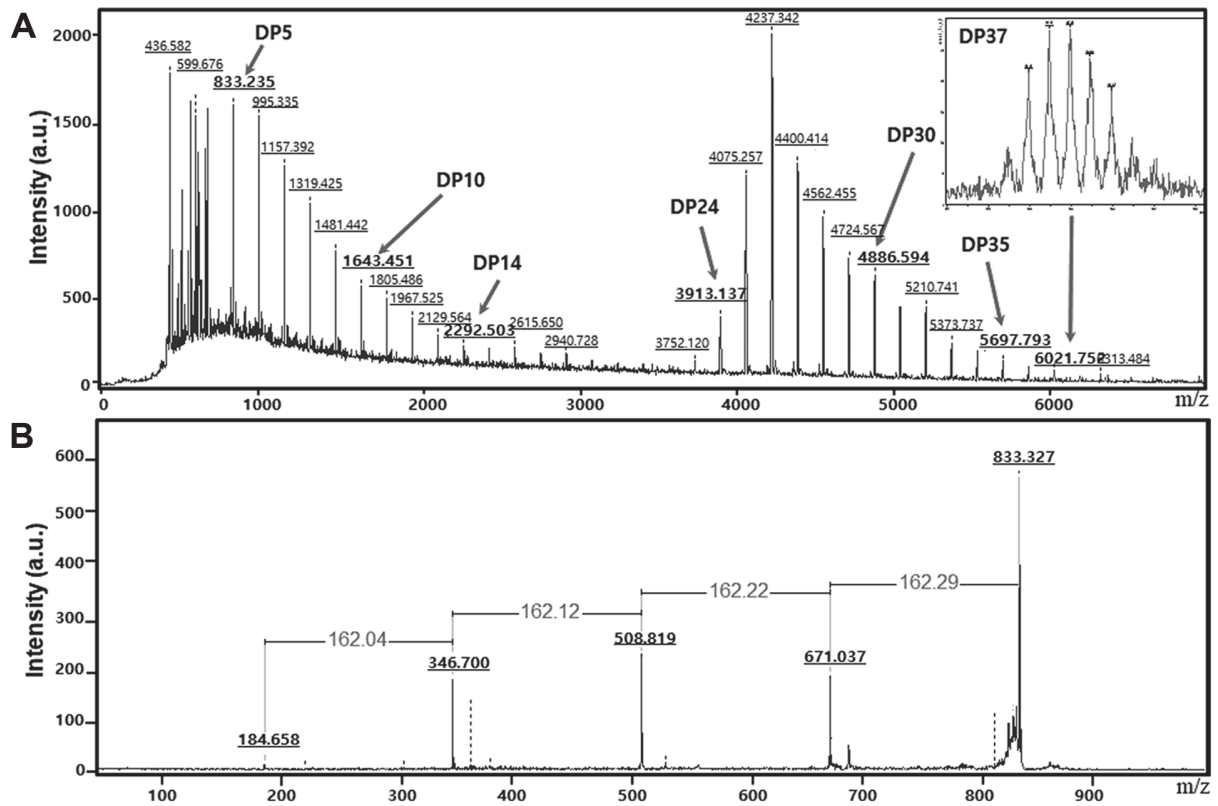

Fig. 5. MALDI-TOF/MS analysis of cycloamyloses produced by DgaGT. The number on each peak indicates the molecular mass (A). CDP5 was generated profoundly. Tandem mass spectrometry analysis reveals that the peak with $\mathrm{Mw}$ 833.235 was CDP 5 product which consists of anhydroglucoses $(\mathbf{B})$.
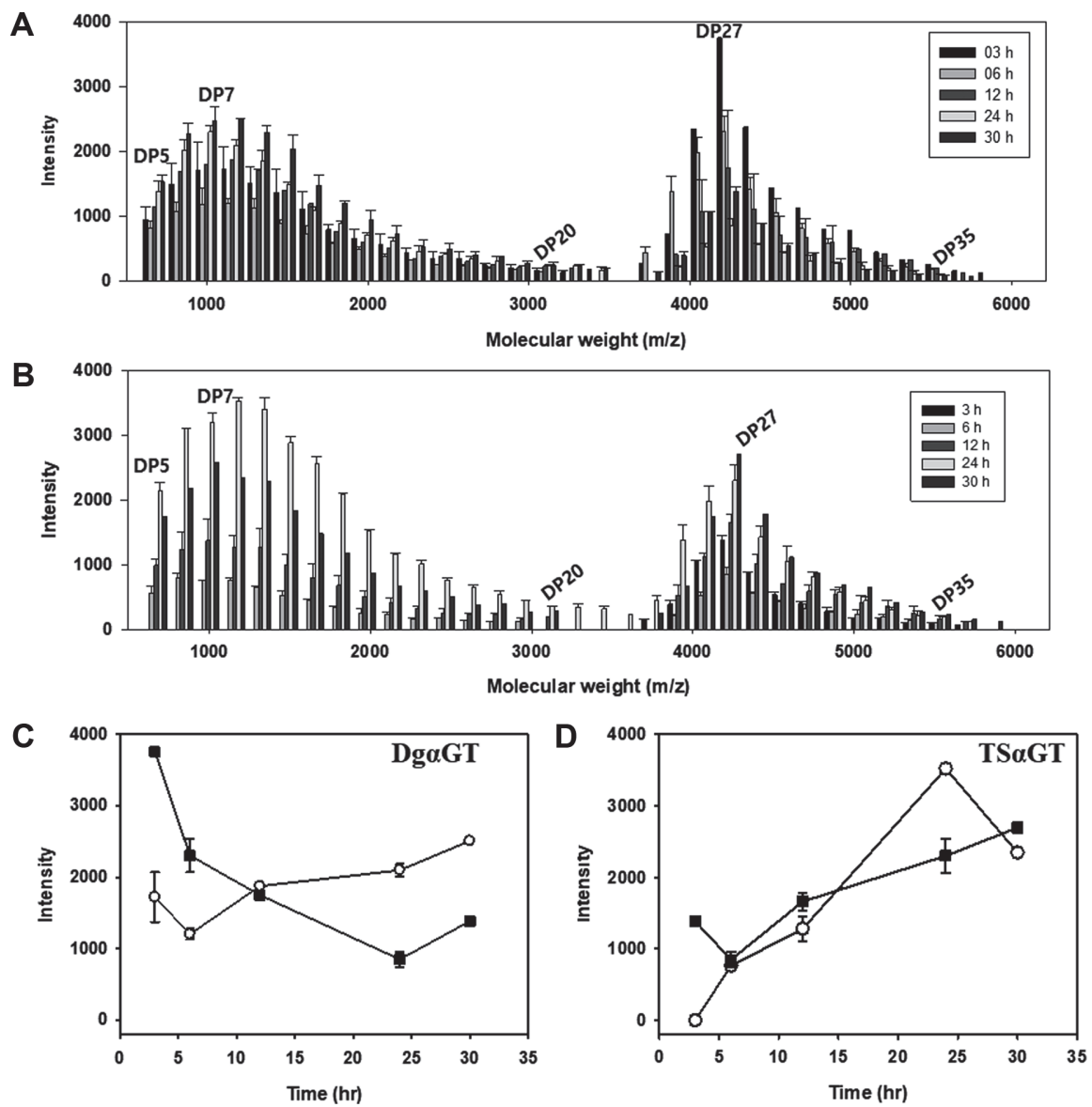

Fig. 6. Composition of DP5-35 cycloamyloses produced by DgaGT (A) and TSaGT (B). Note, no cycloamylose under DP22 was detected at $3 \mathrm{~h}$ reaction sample of TSaGT. Quantitative variation of DP7 (white circle) and DP27 (black square) cycloamayloses during the reaction are compared at (C) and (D). 
was continued to the equilibrium point where no more significant change of products was observed. DgaGT produced a significant amount of CA from amylose, and the maximum yield was about $45 \%$ at 6 h (Fig. 4). Intriguingly, TsaGT showed much slower production and reached maximum after $24 \mathrm{~h}$. The yield of TsaGT was similar to that of DgaGT. Further optimization might be needed to increase the CA production yield by TsaGT.

The CA distribution and peak shapes were similar in MALDI-TOF/MS chromatograms. The bimodal shapes appeared from early stage of CA synthesis for both enzymes. DP 6 8 and DP 27 were detected as peak DPs in the distribution (Fig. 5). The larger DP CA, especially CD 27, quickly decreased as the reaction continued in the CA product from DgaGT, and proportion of the smaller CA increased fast. In TsaGT's case, the small CA increased faster than that of DgaGT but the amount of large CA also, to lesser extent, increased (Fig. 6). The large CA was used as substrates for the coupling reaction in the DgaGT reaction. TsaGT uses large CA for the coupling reaction, too. But the effect was not significant due to the ratio between the small CA and the larger CA at the early reaction stage. The CA distributions showed typical U-shapes with very low amount of the mid-size CA (DP 15 24) (Fig. 6). Steric hindrance by a protruding structure at the middle of the catalytic site of aGTs was suggested as the mechanism of this DP selectivity [25].

Cyclomaltopentaose (CDP5), a rare cyclic glucan, was detected at the early of reaction and accumulated through the reaction. The enzymes produced a large amount of CDP5 similar to the amount of CDs (Fig. 5B). In previous studies, CDP5 with production of all $\alpha$-1,4-linkages was not successfully detected. A computer modeling study reported that the torsion angle in CDP5 was not possible in the enzyme synthesis [26]. However, our results clearly indicate CDP5 production is able to be achieved by DgaGT and TsaGT.

In brief, all three enzymes showed their higher activity in catalysis of disproportionation and coupling than cyclization and hydrolysis. MalQ, thoroughly different in phylogenetic analysis from thermophilic bacteria but profoundly homologous to pathogenic bacteria, had a fairly low cyclization activity in comparison with DgaGT and TsaGT. CA products with bimodal shape of DP distribution were produced similarly by DgaGT and TsaGT, but not really much by MalQ. It is notable that TsaGT did not produce short DP CA at the early reaction stage. Among these CAs, cyclomaltopentaose was formed at early reaction stage and accumulated over the course of time, especially in TsaGT catalysis. This study should provide valuable insight for production of CA with food and pharmaceutical industry applications.

\section{Acknowledgments}

This work was supported by a National Research Foundation of Korea (NRF) grant funded by the Korea government (MSIT) (No. 2015R1D1A1A01058005) and a research fund of Chungnam National University.

\section{Conflicts of Interest}

The authors have no financial conflicts of interest to declare.

\section{References}

1. Nguyen DHD, Park S-H, Tran PL, Kim J-W, Le QT, Boos W, et al. 2019. Characterization of the transglycosylation reaction of 4- $\alpha$ Glucanotransferase (MalQ) and its role in glycogen breakdown in Escherichia coli. J. Microbiol. Biotechnol. 29: 357-366.

2. Jeong D-W, Jeong H-M, Shin Y-J, Woo S-H, Shim J-H. 2019. Properties of recombinant 4-a-glucanotransferase from Bifidobacterium longum subsp. longum JCM 1217 and its application. Food Sci. Biotechnol. 29: 667-674.

3. Takaha T, Smith SM. 1999. The functions of 4-a-glucanotransferases and their use for the production of cyclic glucans. Biotechnol. Genet. Eng. Rev. 16: 257-280.

4. Takaha T, Yanase M, Okada S, Smith SM. 1993. Disproportionating enzyme (4-alpha-glucanotransferase; EC 2.4. 1.25) of potato. Purification, molecular cloning, and potential role in starch metabolism. J. Biol. Chem. 268: 1391-1396.

5. Park J-H, Kim H-J, Kim Y-H, Cha H, Kim Y-W, Kim T-J, et al. 2007. The action mode of Thermus aquaticus YT-1 4-aglucanotransferase and its chimeric enzymes introduced with starch-binding domain on amylose and amylopectin. Carbohydr. Polym. 67: 164-173.

6. Lee KY, Kim Y-R, Park KH, Lee HG. 2006. Effects of a-glucanotransferase treatment on the thermo-reversibility and freeze-thaw stability of a rice starch gel. Carbohydr. Polym. 63: 347-354.

7. Terada Y, Fujii K, Takaha T, Okada S. 1999. Thermus aquaticus ATCC 33923 amylomaltase gene cloning and expression and enzyme characterization: production of cycloamylose. Appl. Environ. Microbiol. 65: 910-915.

8. Liebl W, Feil R, Gabelsberger J, Kellermann J, Schleifer KH. 1992. Purification and characterization of a novel thermostable 4-a-glucanotransferase of Thermotoga maritima cloned in Escherichia coli. Eur. J. Biochem. 207: 81-88.

9. Dippel R, Boos W. 2005. The maltodextrin system of Escherichia coli: metabolism and transport. J. Bacteriol. 187: 8322-8331.

10. Lim MS, Myung Hee Lee, Jeong Huyn Lee, Hyun-Mok Ju, Na Young Park, Hye Sook Jeong, et al. 2005. Identification and characterization of the Vibrio vulnificus malPQ operon. J. Microbiol. Biotechnol. 15: 616-625.

11. Han A-r, Lee Y-j, Wang T, Kim J-W. 2018. Glycogen metabolism in Vibrio vulnificus affected by malP and malQ. Microbiol. Biotchnol. Lett. 46: 29-39.

12. Park J-T, Shim J-H, Tran PL, Hong I-H, Yong H-U, Oktavina EF, et al. 2011. Role of maltose enzymes in glycogen synthesis by Escherichia coli. J. Bacteriol. 193: 2517-2526.

13. van der Veen BA, van Alebeek GJW, Uitdehaag JC, Dijkstra BW, Dijkhuizen L. 2000. The three transglycosylation reactions catalyzed by cyclodextrin glycosyltransferase from Bacillus circulans (strain 251) proceed via different kinetic mechanisms. Eur. J. Biochem. 267: 658-665.

14. Tachibana Y, Takaha T, Fujiwara S, Takagi M, Imanaka T. 2000. Acceptor specificity of 4-a-glucanotransferase from Pyrococcus kodakaraensis KOD1, and synthesis of cycloamylose. J. Biosci. Bioeng. 90: 406-409.

15. Yanase M, Takata H, Takaha T, Kuriki T, Smith SM, Okada S. 2002. Cyclization reaction catalyzed by glycogen debranching enzyme (EC 2.4. 1.25/EC 3.2. 1.33) and its potential for cycloamylose production. Appl. Environ. Microbiol. 68: 4233-4239.

16. Altschul SF, Madden TL, Schäffer AA, Zhang J, Zhang Z, Miller W, et al. 1997. Gapped BLAST and PSI-BLAST: a new generation of protein database search programs. Nucleic Acids Res. 25: 3389-3402.

17. Laemmli UK. 1970. Cleavage of structural proteins during the assembly of the head of bacteriophage T4. Nature 227: 680-685. 
18. Werner W, Rey He, Wielinger H. 1970. Properties of a new chromogen for determination of glucose in blood according to god/podmethod. Z. Anal. Chem. Freseniu. 252: 224.

19. Koizumi K, Sanbe H, Kubota Y, Terada Y, Takaha T. 1999. Isolation and characterization of cyclic $\alpha-(1 \rightarrow 4)$-glucans having degrees of polymerization 9-31 and their quantitative analysis by high-performance anion-exchange chromatography with pulsed amperometric detection. J. Chromatogr. A 852: 407-416.

20. Fox JD, Robyt JF. 1991. Miniaturization of three carbohydrate analyses using a microsample plate reader. Anal. Biochem. 195: 93-96.

21. Oh MJ, Hua S, Kim BJ, Jeong HN, Jeong SH, Grimm R, et al. 2013. Analytical platform for glycomic characterization of recombinant erythropoietin biotherapeutics and biosimilars by MS. Bioanalysis 5: 545-559.

22. Murakami T, Kanai T, Takata H, Kuriki T, Imanaka T. 2006. A novel branching enzyme of the GH-57 family in the hyperthermophilic archaeon Thermococcus kodakaraensis KOD1. J. Bacteriol. 188: 5915-5924.

23. Sasaki Y, Nomura Y, Sawada S-i, Akiyoshi K. 2010. Polysaccharide nanogel-cyclodextrin system as an artificial chaperone for in vitro protein synthesis of green fluorescent protein. Polymer J. 42: 823-828.

24. Yamaguchi H, Miyazaki M. 2014. Refolding techniques for recovering biologically active recombinant proteins from inclusion bodies. Biomolecules 4: 235-251.

25. Gattuso G, Nepogodiev SA, Stoddart JF. 1998. Synthetic cyclic oligosaccharides. Chem. Rev. 98: 1919-1958.

26. Watanabe H, Nishimoto T, Sonoda T, Kubota M, Chaen H, Fukuda S. 2006. An enzymatically produced novel cyclomaltopentaose cyclized from amylose by an $\alpha$ - $(1 \rightarrow 6)$-linkage, cyclo- $\{\rightarrow 6)-\alpha$-D-Glcp- $(1 \rightarrow 4)-\alpha-D-G l c p-(1 \rightarrow 4)$ - $\alpha$-D-Glcp- $(1 \rightarrow 4)-\alpha-D-G l c p-(1 \rightarrow 4)$ a-D-Glcp- $(1 \rightarrow\}$. Carbohydr.Res. 341: 957-963. 